\title{
Internet como una esfera pública y el papel actual del Parlamento
}

\section{Internet as a public sphere and the current role of the Parliament}

\section{RESUMEN}

La popularización de las tecnologías de la información y las comunicaciones (TIC) trajo consigo profundos cambios en distintos ámbitos de la sociedad. Desde el mercado de las instituciones públicas, estas recientes y significativas modificaciones han dividido las opiniones y esperanzas en la superación de las actuales limitaciones impuestas a las interacciones sociales sin estas tecnologías. Hablando de la representación política y la expresión de los valores democráticos, no es diferente; esta divide opiniones acerca del presente y el futuro en lo que respecta a la voluntad política y el ejercicio de la soberanía popular. Este artículo inicia con un breve análisis sobre el concepto de esfera pública, con el objetivo de investigar el uso del Internet considerado un verdadero medio para el logro de una esfera pública global. Esto es confrontado con el problema de la estructura actual de la red, que cada vez se basa más en los intereses privados, lo que impone desafíos teóricos y prácticos para el logro de un verdadero entorno democrático. Por último, todos estos conceptos se utilizan para enfrentar el problema sobre el papel actual de los parlamentos en el proceso legislativo para el funcionamiento de la democracia contemporánea.

\section{PALABRAS CLAVE}

Internet, democracia, proceso legislativo, esfera pública, Parlamento.

\section{ABSTRACT}

The popularization of the Information and Communication Technologies brought deep changes in many areas of society. From the market to the public institutions, these significant and recent behavioral modifications has been dividing opinions and hopes in the overcoming of the current limitations imposed to social interactions without these technologies. Talking about the political representation and the expression of the democratic values it is not different. It splits opinions about the present and the future in regards of political will and the exercise of popular sovereignty. This work starts first on a brief analysis on the concept of public sphere to inquire the possibilities of the Internet to be considered a true vehicle 
for the achievement of a global public sphere. Later, it is confronted with the problem of the current structure of the Net, more and more based in private interests, what imposes theoretical and practical challenges to the achievement of a true democratic environment. Finally, all this concepts are used to face the problem about the current role of parliaments in the legislative process in the functioning of the contemporany democracy.

\section{KEYWORDS}

Internet, Democracy, Public Sphere, Legislative Process, Parliament.

\section{INTRODUCCIÓN}

La transformación condujo a la popularización de la Tecnología de la Información y las Comunicaciones (TIC), especialmente el internet, que cambió mucho la escena de la política actual. Este enorme universo de posibilidades para la concreción de los valores democráticos, como la libertad de expresión y asociación, entre otros, ha sido liderado por un sinnúmero de personas, que sostienen que el ambiente social de hoy trae implícitamente un gran potencial para el desarrollo de las instituciones democráticas.

Sin duda, el uso de la web puede desarrollar la transparencia, la responsabilidad política, y otras cuestiones de base en un sistema efectivamente democrático. Sin embargo, muchas personas tienen dudas acerca de estas afirmaciones, en una tendencia que crece en la doctrina especializada en los últimos años, lo que lleva a algunos a afirmar que la llamada «democracia digital» sería en realidad un mito (Hindman, 2009). En tiempos de "grandes hechos» y revelaciones de enormes violaciones de los derechos humanos a la privacidad en una escala global, parece que la idea de una web libre se está convirtiendo cada vez más en una imagen del pasado, lo que hace cumplir la profundidad del problema científico a resolver (Rosen, 2013).

El objetivo de este artículo, en primer lugar, es discutir sobre algunos de los retos por lo que el internet es considerado una verdadera esfera pública global. Por lo tanto, existe la necesidad de acudir a una corta pero firme doctrina sobre el concepto de esfera pública y sus elementos. Seguidamente, se confrontarán estas declaraciones con la actual estructura de la Web 2.0, revelando algunos riesgos para el ejercicio de la ciudadanía en la actualidad.

La hipótesis es que el rol de la representación política se juega por los parlamentos de los Estados democráticos que parecen estar en peligro, los cuales conducen cada vez más a una gran pregunta: ¿cuál es el rol de la web en la estructura de un Estado moderno?

\section{CONSIDERACIONES BREVES SOBRE EL CONCEPTO DE ESFERA PÚBLICA}

Muchos utilizan la noción de esfera pública o espacio público en la teoría democrática. Sin embargo, la doctrina utiliza a su vez la obra de Habermas (1997) para la comprensión del concepto de una manera más precisa: in verbis.

La esfera pública es un fenómeno social elemental, de la misma manera que la acción, el actor, el grupo o la sociedad; sin embargo, no es uno de los conceptos tradicionalmente utilizado para describir el orden social. La esfera pública no puede ser entendida como una institución, ni como una organización, dado que no es una estructura normativa capaz de asentar las diferencias entre las competencias y sus funciones, ni tampoco en regular la manera de pertenecer a una organización, etc. Tampoco se hace un sistema, a pesar de que sea posible establecer sus límites internos, se caracteriza por fuera de los horizontes abiertos, que son permeables y variables. La esfera pública se puede describir como una red de comunicación adecuada para conocer el contenido, la posición y la opinión; en ella, los flujos comunicacionales se filtran y se sintetizan, en un punto tal que se condensan en las opiniones que 
están agrupadas en temas específicos. De la misma manera que la vida a una escala mundial, la esfera pública se reproduce a través de la actuación comunicativa, lo que implica sólo el uso de un lenguaje natural; sincronizada con la comprensión general de la práctica comunicativa diaria.

Se evidencia que la vida del mundo no es más que un reservatorio para la interacción sencilla; con los sistemas especializados de las acciones del conocimiento, que están formados al interior de la vida del mundo, manteniéndose atados a este. Se conectan a las funciones generales de la reproducción y la existencia global (como la religión, la escuela, la familia), para diferentes aspectos de la validez de los conocimientos que se trasmiten a través del lenguaje común (como la ciencia, la moral, las artes). Sin embargo, la esfera pública no está especializada en ninguna de estas dos direcciones, por lo que, cuando se incluye cuestiones políticas relevantes, deja la elaboración especializada al sistema político.

La esfera pública constituye principalmente una estructura comunicacional de actuar orientada por el entendimiento, que se comunica con el espacio social creado en la actuación comunicativa, no con las funciones o el contenido de la comunicación del día a día. Por tanto, el concepto mezcla elementos negativos y positivos, pero el análisis hecho se limita a sus características fundamentales. Uno de los puntos más destacables es la diferencia entre los «actores» y el "público», en un ambiente donde algunos grupos o individuos influyen más en la opinión de los demás.

De esta manera, la lucha por la influencia revela los aspectos de la llamada sociedad civil, que mezcla elementos de lo público y lo privado en el rapto de opiniones favorables a las pretensiones de uno $u$ otro grupo de personas. De acuerdo a Habermas (1997), hoy en día, se juega un papel decisivo por el significado de la comunicación masiva, importan- te en este entorno en la formación de la llamada opinión pública.

Por último, esto debe ser independiente de la disposición institucional, lo que Dahl (2012) denomina como la segunda generación de la democracia ${ }^{1}$, cuyas estructuras están todavía muy presentes en la opinión pública, la cual tiene una gran influencia en el Estado, dirigiendo los debates políticos para las elecciones, aunque no solo elecciones. Por lo tanto, los debates en el espacio público que crean la opinión pública, deberían tender a reflejar en la composición del Parlamento, y en todas las instituciones que integran su formación (formales o no), como los partidos políticos e intereses grupales.

Este artículo no pretende reducir la pesada obra de Habermas y su formulación del concepto de esfera o espacio público en unas pocas líneas. Sin embargo, el concepto es de crucial importancia para la dosificación de las potencialidades y límites que la web tiene hoy en día como una forma de superación del concepto de representación política y de sus artimañas jurídicas, como las elecciones periódicas, el voto, la representación política, etc.

\section{LA WEB COMO UNA ESFERA PÚBLICA GLOBAL}

Después de establecer algunas nociones sobre el concepto de la esfera pública en la obra de Habermas, es notable que se trata de los limites generales, en una red de comunicación en donde los «actores» y el «público» se mezclan como jugadores, buscando la influencia y, naturalmente el poder político. De esa manera, la aparición de las TIC, traerá implícitamente, unas alteraciones sensibles tanto en su estructura como en su área.

Por ello, existen y han existido muchas voces que identifican la web como un espacio público real, apto para el debate franco y abierto de ideas, donde se puede ejercer por completo las libertades de

1 A pesar de la extraordinaria influencia de la Grecia clásica en el desarrollo de la democracia, las ideas y las instituciones democráticas modernas también están conformados por muchos otros factores, de los cuales tres son de particular importancia: una tradición republicana, el desarrollo de los gobiernos representativos y ciertas conclusiones que tienden a venir. Dahl (2012). 
expresión, comunicación e información en las perspectivas activas y pasivas. De esta manera, para Castells (2013) la internet es un factor determinante para la formación de una esfera pública global, donde los espacios de la Web 2.0, como los blogs y las redes sociales juegan un papel fundamental.

Esta esfera pública global se construye cerca del sistema de medios de comunicación y la web, en particular en los espacios sociales de la Web 2.0, como YouTube, MySpace, Facebook y la blogosfera en crecimiento, que en 2007 llegó a 70 millones de blogs, duplicando su tamaño cada seis meses (Tremayne, 2007).

Por lo tanto, la estimulación de la consolidación de esa esfera pública basada en la comunicación es una herramienta clave en que los Estados y las instituciones internacionales pueden desarrollar de alguna forma las demandas y proyectos de la sociedad civil. Eso puede ocurrir si el diálogo sobre la iniciativa específica y la consolidación de forma continua, de las contribuciones a este diálogo, la aplicación de la formulación de políticas públicas en el campo internacional se estimulan. Usando el poder de la opinión pública en todo el mundo a través de redes de comunicación globales y del internet es la forma más eficaz de ampliar la participación política en una escala global, inducir a una buena conexión entre las instituciones gubernamentales e internacionales en una sociedad civil global. Este espacio de la comunicación multimodal es lo que constituye la nueva esfera pública global (Castells, 2008).

Según (Castells, 2013), afirma que corrobora su tesis mediante el análisis de la forma social, puesto que las redes contribuyeron para la eclosión de una serie de manifestaciones populares, como la marcha iracunda en España (2011-2012), la llamada primavera Árabe en muchos países de Oriente Medio (2011-2012) e incluso las protestas en Brasil que comenzaron con el movimiento "Passe Livre», en São Paulo, pero se extendió por todo el país durante la Copa Confederaciones de la FIFA.

Otros autores también revelan un gran entusiasmo con el potencial no sólo del internet, sino de las TIC, para la formación de un medio ambiente en todo el mundo ciberdemocrático. De esta manera, Lévy y Lemos (2010) afirman que:

La opinión pública (y con ella los lugares comunes, los prejuicios y todo tipo de opiniones de sentido común) es cada vez más global. Con los teléfonos móviles multifuncionales (cámara, correo electrónico, SMS), la capacidad de producción de la información desde cualquier lugar (y por cualquier persona) hace la visibilidad global más precisa (p.158).

A pesar de Lévy y Lemos (2010) insisten en que, por ejemplo, la ausencia de la intimidad en la sociedad hoy en día es un enorme riesgo para la prodigalidad de estas ideas democráticas, acaban de concluir que la democracia se ha globalizado en sí, frente a la "globalización del capital», y será revelado en tres grandes frentes: i). La promoción de experiencias democráticas locales; ii). La formación de un gobierno mundial; iii). El desarrollo de las virtudes civiles que implican consumo, inversión y trabajo.

De la misma manera, Maia (2008), señala que «la web no puede ser tomada como una esfera pública». Sin embargo, expresa:

(...) la comunicación de información, que se despliega libremente entre los individuos y las comunidades, fuera de los espacios políticos formales, debe ser vista como un recurso importante para una interpretación productiva de las preocupaciones políticas y las formulaciones de la demanda que se enviarán a los órganos administrativos y deliberativos del sistema político (p. 281).

En otras palabras, Maia (2008) afirma que la web interviene en el espacio público, donde se pueden debatir cuestiones de interés público, pero interactúa con los órganos deliberativos, como el Parlamento. Es decir, la web tomaría parte del proceso que compone la democracia deliberativa, pero no es el lugar de la deliberación en sí. Al menos por ahora, ya que hay dos planes distintos en la democracia deliberativa: i). El proceso informal de la voluntad de la constitución en la esfera pública; ii). La deliberación política que es regulada por procedimientos típicos de los sistemas políticos específicos (p. 280). 
Vaidhyanathan (2012), manifiesta que la obra de Habermas influencia en los esfuerzos para una reformulación de los medios de comunicación y -en una extensión muy corta- la política de medios de comunicación. Cansada de reconstruir el griego «Ágora», debería tratar de construir mejores cafés» (pp. 136-137), refiriéndose al famoso texto del autor en la que se le atribuye a las cafeterías de Europa el papel de la formación de la opinión pública en la sociedad burguesa.

No obstante, después de eso, Vaidhyanathan (2012) critica no sólo el propio concepto de esfera pública, que culpa por el fracaso de ser restringida histórica y geográficamente, como la afirmación de que la web de hoy se puede comparar con la «sociedad civil cosmopolita, o su pariente, la esfera pública global. En virtud de lo anterior, es posible resaltar: i). La exclusión digital, reforzada por el hecho de que, a pesar de que el número de usuarios de la web está creciendo formalmente, la cantidad de redes de alta velocidad (que da acceso a contenidos más sofisticados, tales como YouTube) es todavía pequeña; ii. La falta de habilidades para participar en la sociedad civil globalizada y tecnológica, lo que conduce a un aumento de la diferencia entre los «actores» y el «público», ya señalado por Habermas.

Sin embargo, la piedra de toque en las observaciones del Vaidhyanathan es acerca de la actuación de un actor específico en la actual web y los riesgos para el papel de las discusiones políticas dando lugar a la misma: Google. Para quien, la aplicación del concepto de relevancia en los filtros de las herramientas de búsqueda, influye en la personalización de la información que llega al final al usuario/consumidor, interviniendo fuertemente en la formación de la opinión y, naturalmente, en la estructuración de un proceso abierto para la formación de discusiones políticas y de opiniones públicas.

Mientras tanto Google continúa buscando personalizar y particularizar sus servicios y resultados, el sentido del conocimiento común siendo su prioridad encontrar sus falencias. Todo puede estar disponible para todo el mundo [...], pero la información esencial puede estar bien clasificada en las investigaciones, aduciendo que es hecho en Sydney, cuando en realidad fue hecho en Sao Paulo. Cuando sólo hay un proveedor de aplicaciones de Internet con un gran tráfico de información, no es difícil decir que tiene una gran influencia sobre una red, la cual se supone que nació para ser descentralizada. Sin embargo, el autor dice en el lanzamiento de su obra, que el problema no es Google, sino ¿Qué tanto y por qué usamos esta y otras aplicaciones de Internet, como Facebook y otros, que concentran un número aterrador de usuarios que también puede tener una influencia profundamente negativa en la esfera pública? (Vaidhyanathan, 2012:139).

Como bien se sabe, la web se basa ahora en la llamada estructura de la Web 2.0. Esto es un slogan para hablar de un grupo de modelos de negocio de los proveedores de aplicaciones en las que el usuario produce el contenido y esta información es el principal capital del empresario y la creación de un medio más eficiente para el editor, ya que señala el anuncio directamente a los consumidores preferenciales (O'Reilly, 2013).

Por lo tanto, en este entorno se produce mucha más información de lo que es posible consumir. Por ello, muchos profesan la superación de la «sociedad de la información» y el apaciguamiento de la «sociedad relevante», en la que hay una falta de precaución. De esta manera, Pariser señala:

Las empresas se dieron cuenta de que colapsó la precaución que se mantenía, como las opciones de información el número de personas aumentó cada vez más. Los que querían beneficiarse deberían recibir atención de la gente. $Y$ en un mundo donde hay una falta de atención, la mejor manera de hacer esto, sería proveer un contenido que en realidad pueda asistir a sus intereses, deseos y necesidades particulares de las personas. En las salas y centros de computación de Silicon Valley, hubo una nueva palabra más importante: relevancia (p. 27).

Especialmente para el Derecho, la aparición de la llamada «burbuja de filtro» puede afectar sensiblemente el poder de la persona elegida, restringiendo algo 
que es parte de la base de la ciencia jurídica: la autonomía privada. Tanto en el derecho público como en el privado, en el contrato o en la elección de electores, la tecnología puede limitar mucho el ejercicio de la libertad que le da la base axiológica para la primera dimensión de los derechos fundamentales.

En cuanto al ejercicio de la ciudadanía, Pérez (2012) de acuerdo a Sustein (2007), señala que es posible identificar dos perspectivas de la ciudadanía en este entorno tecnológico. La primera es la Ilamada Ciberciudadanía, que revela aspectos positivos innegables de la utilización de la tecnología para el acceso al conocimiento, la información, y mejorar la honestidad, entre otros ${ }^{2}$.

Sin embargo, como se desprende Sustein (2007), la web y sus normas basadas en modelos privados de apropiación terminan creando un ambiente que se supone que es público, pero, en el fondo proviene de un simulacro, «republic.com», cuyo modelo de la ciudadanía es la de «citizenship.com». En términos generales, son ciudadanos cuya conciencia cívica es movida por la información que consumen, marcada por la pasividad.
Analizando las perspectivas de la democracia en la sociedad de la información, Bernardes (2013) distingue dos actitudes antagónicas: optimistas y pesimistas. Entre los primeros, Castells (2008) y Levy (2010). Entre los otros, Lessig (1999) y Bauman (2013). A pesar de que parece superficial adoptan el «optimismo frente al pesimismo» como criterios para categorizar los enormes libros analizados, Pérez (2002) también adopta un factor de clasificación similar cuando los atributos de Sustein (2013) se etiquetan de «pesimistas» sobre la democracia de la web, lo que parece ser su propia opinión sobre el tema (Trein, 2013).

Sin embargo, para direccionar el problema de las perspectivas para el Parlamento y la democracia misma, frente a las TIC, no sólo basta con tener «optimistas» 0 «pesimistas». De hecho, Zagrebelsky (2011) busca una vía Tercis para analizar dogmas democráticos. Es la llamada democracia crítica, entre la actitud escéptica y dogmática. Al final, concluye que la actitud jurista hacia transformaciones sociales debe ser el espíritu de la posibilidad, no se le da a un optimismo ciego, ni a un pesimismo estúpido. En verbis:

2 Es importante separar los puntos elegidos por el Manifiesto del ejercicio de una ciberciudadanía activa, responsable y comprometida. Dicha declaración fue elaborada por el 1er Congreso Online del Observatorio para la Ciber-Sociedad (2002):

i) Proclamación de la libertad e igualdad del ciberespacio. Se propugna una apuesta decidida de los gobiernos y los organismos internacionales para el progresivo establecimiento de las infraestructuras y medidas necesarias que brinden a todo ser humano la posibilidad de ejercer su ciberciudadanía, con lo que se vaya reduciendo primero y erradicando después, la fractura digital (punto 1). Asimismo, se auspicia la creación de un marco legal que permita la libertad de servicios en el ciberespacio sin barreras ni proteccionismos, que perjudiquen a persona o sociedad alguna (punto 2). Se defienden las ventajas derivadas de utilizar soluciones tecnológicas de código libre en las administraciones públicas y su implantación, siempre que sea viable, en detrimento de herramientas de tipo comercial, privado o cerrado (punto 4). Se propugna, además, una política tendente a velar por la libre y fluida difusión de la información y el conocimiento en formatos tecnológicos públicos, que permitan que estos recursos sean fácilmente localizables y utilizables (punto 6).

ii) Fortalecimiento de la cultura cívica. Los propulsores de la ciberciudadanía vinculan su plena eficacia a la elaboración, y realización de programas educativos a todos los niveles, también de cultura cívica, que propicien la utilización de las TIC y que «permitan que su utilización y provecho no quede limitado a los grupos social y económicamente privilegiados» (punto 5).

iii) Estrategias de tutela de la ciberciudadania. Los autores del Manifiesto abogan por la implantación de organizaciones que protejan jurídicamente la ciberciudadania contra las prácticas abusivas de gobiernos o empresas que afecten aspectos de fondo, forma, cualitativos o cuantitativos de sus derechos (punto 3). Se proponen también: «la denuncia de incumplimiento de los puntos contenidos en este manifiesto con especial referencia de las consecuencias sociales a las que dicho incumplimiento nos lleva. Convirtiéndonos, de facto, en una voz firme que remueva las conciencias de las personas y de las administraciones públicas» (punto 7). Por último, los signatarios de la declaración, conscientes de la facilidad de hacer público todo tipo de información a través de la Red, se comprometen a promover la elaboración y defensa de los contenidos que puedan servir de guía, referencia o información para reforzar la ciberciudadanía; desde un ejercicio de responsabilidad ética, que tome en consideración las posibles consecuencias de la información publicada. Entienden quienes han formulado el manifiesto que: «ya no es posible seguir poniéndole vallas al mundo, ni fronteras, ni aduanas, ni peajes monopolísticos u oligárquicos. Porque estas vallas, fronteras, aduanas y peajes son testigos de un mundo caduco e injusto al que debemos renunciar para que la Humanidad sobreviva y porque creemos que el ciberespacio es el primer lugar donde esos obstáculos pueden ser, efectivamente, salvados» (punto 8). (Pérez, 2002. pp. 101, 115, 121). 
El punto de actitud dogmática y escéptica común es la imposibilidad de enfrentar lo que puede suceder y la capacidad de no dar marcha atrás, incluso frente a lo que es irreparable. Por lo tanto, se les llama democracia dogmática y escéptica. En el espíritu implacable de la necesidad, el pensamiento de la verdad y la fuente del poder son aliados. En este punto, estos pensamientos son radicalmente diferentes del espíritu de la posibilidad de enfatizarse en un camino diferente, y de concebir la democracia, una democracia que llamaremos democracia crítica [...]. En esta doble actitud, abierta para el optimismo, pero no cerrada para el pesimismo, no es el punto de equilibrio de la democracia crítica. La aspiración para lo mejor, no sacudida por el miedo a lo peor, haría un constante statu quo de superación la cual comenzaría con una frustración social $\mathrm{y}$, dentro de ellos, las demandas de certidumbre y seguridad, que no son compatibles con la democracia crítica (Zagrebelsky, 2011:132).

Esta es la actitud hacia el problema del internet que se considera hoy en día como una esfera pública global, capaz de reemplazar todas las formas tradicionales de representación política y sus estructuras jurídicas en un breve periodo de tiempo. Sin abandonar el sueño de una democracia directa, pero tomando el riesgo de las tecnologías en la cual la humanidad es cada vez más dependiente y, al mismo tiempo, desarrolla y gestiona de acuerdo a los intereses de pocas empresas poderosas.

\section{ACTUALES PERSPECTIVAS LEGISLATIVAS: ¿ES POSIBLE SUPERAR EL PARLAMENTO COMO EL CENTRO DEL PROCESO LEGISLA- TIVO?}

La vinculación de la información y la comunicación con las afirmaciones democráticas, ya expuestas, mejoró considerablemente las posibilidades que desean las manifestaciones populares. Por lo tanto, no hay falta de voceros que profesen el entorno que hoy se manifiesta al estar lleno de experiencia cuya «eliminación intermedia», también es la política del Estado. Sin embargo, como también se dijo, el reconocimiento de las potencialidades no puede ocurrir sin medir los riesgos.
La sustitución de productos intermedios es una vieja promesa de la web. La eclosión de un entorno tecnológico descentralizado y neutral aún lleva a muchas personas a la conclusión de que será la fuerza que moverá la democracia participativa en el futuro, superando la influencia negativa sobre la esfera pública anteriormente, ejercida por los medios masivos de comunicación, como la televisión y la prensa. Sin embargo, para Pariser, apoyado por Tim Wu, es una promesa no remunerada, como el internet no ha eliminado los intermediarios, sólo los reemplazó:

[...]A pesar de que la sumisión a los mediadores son un problema real, la historia de desintermediación es muy mitológica. Su efecto real es hacer nuevos mediadores invisibles. "Es que muchos se disputan el poder», afirma la revista Time cuando anunció que «usted» fue una persona del pasado. Sin embargo, en las palabras de Tim Wu, profesor de Derecho y escritor del interruptor principal, «el crecimiento de las redes no ha eliminado los intermedios, pero sólo los reemplazó». Y a pesar de que el poder se ha desplazado hacia los consumidores, ya que tienen un número muy grande de opciones sobre los medios de comunicación que consumen, todavía no tienen el poder (p. 58).

La cuestión también se instala a la representación política y, por supuesto, a su lugar tradicional en las democracias contemporáneas: el Parlamento. Especialmente cuando es posible verificar que los medios tradicionales de representación son cada vez más anacrónicos, lo que lleva a muchos a concluir que se trata de una crisis de representatividad política, o simplemente, un enorme déficit democrático entre la voluntad del representante y del representado.

Antes de enfrentarse al complejo problema de la necesidad o no de un Parlamento de hoy, es necesario saber lo que es su deber en el estado actual. Las funciones del Legislativo son sintetizadas así por Carvalho (2009):

a) Representante, lo que significa la capacidad única del Legislativo en la simulación de mosaico social, protegiendo las minorías dándoles 
voz y voto en la producción de leyes. El más importante, según el autor, a la que se le atribuye el carácter de "preliminar», de los cuales descienden todos los demás.

b) Movilizar el poder popular, promoviendo la articulación entre la ejecución de las políticas públicas y el deseo popular.

c) Obstruccionista, fraccionando el poder Ejecutivo, ya que es un órgano el cual hace relaciones, lo que hace que sea más conservacionista.

d) informativa, revelar hechos orientados a acortar la distancia entre los ciudadanos y sus representantes.

e) Educativa, alcanzado por su mayor visibilidad y el atractivo de las cuestiones morales y éticas.

f Electivo, típica del parlamentarismo, en el que el Parlamento elige al primer ministro.

En Brasil, el Congreso tiene casi todas las funciones en el sistema constitucional, esta última, la función electiva. Sin embargo, también hay voces que verifican la falta de eficacia en su actuación.

Esto se debe a la crisis de representatividad de modo recurrente entre la doctrina, lo que hace que surjan preguntas, tales como: las dificultades para el ejercicio de la iniciativa popular; la composición de un grupo gubernamental para evitar el ejercicio de la función obstruccionista (y los otros); la formación de los grupos de presión de todas las partes que actúan en función de sus propios intereses (grupos rurales, grupos de "cristianos», etc.); la falta de información de los Poderes Legislativo y la evidencia del Poder Ejecutivo; y muchos otros factores que llevan a decir que, hoy, el Legislativo está más cerca de ser un problema que una solución a la brecha de representatividad.

Por lo tanto, muchos preguntan si la solución para el problema sería el final del parlamento, Dahl (2012) dice que no lo es. En lo que él llama «poliarquía III», en una manera de superar la primera y segunda generación poliarquías de la democracia, enumera algunas posibilidades que surgen especialmente por la popularización de las TIC, expresando:
Es importante pensar, sin embargo, que el deber de estas innovaciones técnicas no es simplemente hacer más fácil la participación, según lo propuesto por algunos de los que defienden a la democracia participativa. (...). La tecnología en la transformación necesariamente debe ser utilizada de una manera, para bien o para mal. Se puede utilizar para dañar los valores y el proceso democrático o para promoverlos. Sin ningún esfuerzo de conciencia para el uso de la nueva tecnología de la comunicación para la democracia, que puede ser utilizada para objetivos perjudiciales de la misma.

(...) ¿Las élites políticas podrían explorar la tecnología de comunicación interactiva para tirar de las cuerdas de los ciudadanos a trabajar por los objetivos de sí mismos? ¿Podría la nueva tecnología ser una invitación abierta para que las élites políticas consoliden su posición en el casi guardián de la esperanza que esta nueva tecnología ofrece? (p. 542).

Con estas dos premisas, él expresa que un determinado país podría incluso crear una especie de "consejo permanente», un pequeño grupo de ciudadanos elegidos para ayudar a la toma de decisiones políticas, a la que llama minipopulus. «Los miembros de una minipopulus podrían encontrarse a través de las telecomunicaciones». Señala, sin embargo: "Veo la institución de la minipopulus en poliarquía III no como una sustitución de los órganos legislativos, sino como un complemento. Sería entonces suministrar, no sustituir a las instituciones de poliarquía I y II»(g.n.) Por lo tanto, el Parlamento se mantendría vigente en la visión de Dahl.

Por otro lado, hay quienes sostienen que su actuación en el proceso legislativo lo hará más y más interactivo. Esta es la opinión de Faria (2012), uno de los que pensó en el proyecto de "democracia» del Congreso brasileño. Para él, hay dos tipos de experiencias en la democracia, la institucional y la no institucional. El análisis de experiencias interactivas promovidas en el Legislativo, concluye que a partir de la simbiosis entre los principios de la democracia representativa y los clásicos de democracia participativa, se eleva la interactividad digital en el Parlamento. 
Para promover este análisis, la investigación siguió la simbiosis entre los principios de la democracia participativa y deliberativa y los institutos clásicos de la democracia representativa. Es por esto que es importante saber cómo el parlamento participativo es más legitimado para la elaboración de leyes, incorporando inteligencia colectiva al proceso de toma de decisiones, haciendo la actuación parlamentaria más transparente. A pesar de que el desarrollo de la rendición de cuentas significa para la representación política uno de los valores básicos del régimen democrático clásico, estos tres principios representan grupos relevantes de una visión de la democracia contemporánea con dosis especiales de participación popular que contribuyen para el desarrollo de los déficits democráticos.

Por último, también es posible decir que el Parlamento debe preservar y fortalecer su poder de supervisión sobre los otros poderes, especialmente el Ejecutivo. Sin embargo, su papel como centro del proceso de elaboración de leyes será paso a paso sustituido por otros dispositivos, y el poder de los dispositivos no institucionales de participación y manifestación de la voluntad popular debe ser mejorado. En este aspecto, la web se vuelve mucho más relevante.

\section{UN BREVE ESTUDIO DEL CASO DEL PRO- VEEDOR DE SERVICIOS DE LA WEB. LA RESPONSABILIDAD CIVIL EN EL MARCO CI- VIL DE LOS USUARIOS DE INTERNET}

Antes de entrar específicamente en el tema de la responsabilidad por el contenido puesto por otros, algunos aspectos puntuales de la estructura brasileña de derechos civiles para los usuarios de internet se deben exponer brevemente, con el fin de ilustrar sus bases axiológicas; 0 , al menos, las declaradas por la ley.

En primer lugar, el principio lógico y enunciativo de los derechos civiles es un contrapunto a las iniciativas, que antes de puntuar a cuales son los agentes de la Web, intentaron criminalizar los comportamientos de los usuarios, sobre todo para defender los intereses patrimoniales (Longhi, 2009).
El texto legal anuncia como fundamentos: i). El reconocimiento a escala mundial de la web; ii). Los derechos humanos, el desarrollo de la personalidad y el fruto de la ciudadanía en formas digitales; iii). La pluralidad y la diversidad; iv). La apertura y colaboración; v). La libre empresa, la libre competencia y protección del consumidor; y vi). La finalidad de la web social (artículo segundo).

En cuanto a los principios, que se enumeran en una lista ejemplificativa: i). Garantía de la libertad de expresión, la comunicación y la manifestación de pensar, de acuerdo a la Constitución; ii). La protección de la intimidad; iii). La protección de los datos personales, como lo establece la ley; iv). La preservación de la garantía de la neutralidad de la web; v). La preservación de la estabilidad, la seguridad y la funcionalidad de la web, a través de actos técnicos que sean compatibles con las normas internacionales y a través de la estimulación del uso de buenas prácticas; vi). Responsabilidad de los agentes de acuerdo con sus actividades bajo los términos de la ley; y VII. Preservación de la naturaleza participativa de la web (tercer artículo).

Una cosa que debe ser separado es la inclusión, en el texto sustitutivo propuesto por el relator de la Comisión Especial, para el desarrollo de la personalidad y el objetivo social de la web en las listas respectivas, más allá de la falta de delegación en la tardía regulación de la materia de la neutralidad de la web.

Por otra parte, la finalidad principal de la ley es garantizar la privacidad del usuario. Según Rodotà (2008), el «cuerpo», en la era de la información, no es sólo el cuerpo físico, sino que abarca principalmente el conjunto de los datos personales de los individuos, principalmente los llamados datos sensibles. Estos son parte de nuestro «cuerpo electrónico» y, así, la falta de tutela especial porque representa un aspecto especial de la dignidad humana (p. 17).

El marco brasileño de derechos civiles para los usuarios de Internet también tiene como objetivo evitar la mala práctica de vigilancia que en la actualidad, forman parte de la estructura del modelo de negocio de muchos proveedores de rehenes y, sin embargo, 
a quienes la ley prefiere llamar genéricamente «proveedores de aplicaciones de Internet».

Ubicando las líneas generales, se llegó al punto más controversial propuesto por el texto. Es el sistema de responsabilidad civil consagrado por la ley, en el supuesto de ser convertido en favor de la protección de la privacidad del usuario.

Después de todo, es posible identificar que la estructura de la web ha estado sufriendo alteraciones en los últimos tiempos. En el comienzo de la década de 2000, los modelos de negocio en la Web comenzaron a basarse en el desarrollo de plataformas que podrían permitir la inserción de contenidos por los usuarios, que ha sido nombrado por el lema web comercial 2.0 (O'Relly, 2006).

La remuneración de los intermediarios se hace no sólo por los anuncios en las pautas y enlaces patrocinados, sino principalmente por la llamada publicidad dirigida, basada en los catastros de los consumidores realizados por las preferencias del usuario. Esta comercialización cruza el mercado que va más allá de las pautas y enlaces patrocinados, pero transcurre por toda la estructura de la Web, que se basa en el contenido introducido por los usuarios como capital principal para ser explorado por los intermediarios.

En primer lugar, es importante destacar que la jurisprudencia brasileña ha introducido una sentencia básica en la aplicación del Código de Defensa del Consumidor a los proveedores de rehenes y el contenido de la remuneración indirecta que caracteriza la relación entre proveedores y consumidores.

Esta es la razón por cual la orientación predomina en la Corte Suprema de Justicia, en la actualidad, se inicia desde el punto de construcción, en la jurisprudencia, un sistema que se acerca a la notificación retirada, prevista en la ley extranjera - especialmente americana y europea.

Sin embargo, la falta de un tratamiento especial, sobre todo por los riesgos que implican la tutela de los derechos personales de los usuarios, es la situación de algunos intereses especiales, como los de los niños y adolescentes, que ya han sido objeto de especial atención por parte de los tribunales y las políticas legislativas, como la del artículo -A 241a, $\S 2^{\circ}$, del Estatuto Niño y del Adolescente, que enjuicia penalmente al responsable de mantenimiento en su sitio de contenido relacionado con la pedofilia, si oficialmente se notifica, no se procederá a su retiro efectivo.

De igual modo, el objetivo de la tutela de intereses similares es del Tribunal Superior de Justicia que ha decidido, mantener la medida cautelar que determinó no sólo el bloqueo de las comunidades en el sitio web de Orkut en el que el material ofensivo a los niños y adolescentes (uno de ellos víctima de un delito sexual) se mostró, como la obligación de evitar la creación de otros nuevos con el mismo objetivo. Esto, independiente en una parte significativa de los Tribunales del Estado, que han producido decisiones que utilizan el objetivo y la responsabilidad solidaria de los proveedores más allá de los casos de pedofilia en internet.

Sin embargo, el «marco brasileño de los derechos civiles para los usuarios de Internet», en un principio, trató de introducir un sistema diferente. El artículo 14 entre otros traen consigo la necesidad de la notificación judicial al proveedor para expulsión de cualquier contenido. Además, se dice que el proveedor podrá, si así lo solicita el usuario introducir la información que es objeto de la decisión judicial, sustituyendo el contenido por las razones que lo motivaron.

El sistema utilizado tiene por principio, en las palabras del relator de la sustitutiva, la inimputabilidad de la web (Proyecto de Ley No 2126 de 2011). Las razones de esta opción legislativa fueron puestas por él, manifestando que «esta conducta tiene como objetivo proteger a los muchos intermediarios que son responsables solo por la transmisión y conducción de los contenidos», afirmando que: «la responsabilidad por las infracciones eventuales que provienen del contenido puesto por otros es del que lo cometió, no de quienes protegen infraestructura necesaria para el tráfico de información en internet». 
Por otra parte, se dice expresamente que el sistema aporta una garantía a la «responsabilidad equivocada de los intermediarios en internet», protegiendo "las potenciales innovaciones de la web», excepto por orden judicial con determinación específica. Defiende que el sistema pretende evitar «genéricas órdenes de supresión de contenidos, con la obligación de que la orden judicial sea clara y específica, lo cual es el contenido prohibido, lo que deberá permitir la localización correcta del material».

Por último, se desprende que la sustitución incluida en la nueva redacción de la ley en mención consagra la protección de la libertad de expresión, con el propósito de «evitar la censura, expresando la preocupación de mantener la web como un espacio de libertad», tal como hace hincapié en que «la responsabilidad de que el "caput» de los artículos señalados tienen un carácter civil». Por último advierte que debe hacerse acerca de los riesgos del sistema adoptado a la tutela de los derechos personales del usuario.

La primera es acerca de la necesidad de indicar el lugar específico de información en la Web. Si hay cualquier daño a la personalidad perpetrado por la Web, es común que la información se multiplique rápidamente; es por esto que el usuario debe realizar la petición de retirar las direcciones URL que se encuentran y que están en la web en ese momento. Esta es la razón por la cual Corte Suprema de Justicia ha decidido que el administrador de la página web tiene el deber técnico de evitar la divulgación de contenido prohibido, y no imponer a él la difícil tarea de precisión indicando las direcciones URL (RESP 1175675/ RS, Solomon, L).

Por otra parte, un segundo punto está separado en la justificación del tratamiento legal dado a todos los proveedores de aplicaciones, aduciendo que la falta de responsabilidad es un corolario del derecho fundamental a la libertad de expresión. La libertad de expresión no debe ser absolutamente considerada como si fuera el único valor custodiado por la Ley. Sobre ella, Rosely y Gomes (2008) expresan:

En el momento el discurso de emancipación de la web, fue entendido como una reserva ambiental sin la custodia de alguna medida cautelar y se dedicó a cultivar la plena libertad de expresión. Libertad que, de forma automática, debe ser considerada como una virtud democrática. El modelo de la democracia liberal-individualista conocido como el liberalismo se encuentra en los cyber-anarquistas y sus alcances. Sin embargo, se encontró rápidamente que la ecuación que dice que la libertad está siempre ligada a la democracia y su control junto con la tiranía es más que un dispositivo de la retórica del liberalismo en su forma más extrema. No es malo, peligroso, criminal, $u$ ofensivo para la dignidad humana, ni perjudicial a la información antidemocrática el defender su derecho a existir, no es lo mismo que la lucha de los derechos civiles en el ciberespacio. Por el contrario, puede significar la contratación en el discurso del odio, el racismo público, la protección de la discriminación de las minorías. Si en la web realmente nace la libertad de expresión y el espacio para experiencia democrática, se ha convertido en el paraíso de los conservadores, la ultra derecha política, los racistas y la xenofobia, un refugio que, por cierto, ha sido más seguro y más rico para el mundo fuera de línea (pp. 321-322).

Las leyes sobre la responsabilidad del proveedor por el contenido generado por los demás tienen su escritura original que cambió dos veces. Un primer momento, antes de la elaboración del proyecto de ley que se envió por el Ejecutivo, dando al proveedor la obligación de mantener en su sitio web, un canal para recibir notificaciones y contra notificaciones de los usuarios que quieren retirar el contenido prohibido, en un sistema similar al utilizado en el extranjero. La escritura original también igualó a los usuarios que dirigieron páginas de divulgación de contenido y tenían el control de esta información a los proveedores a ese objetivo.

El sistema creado ha sido sustituido por el que se utiliza ahora, que tiene la necesidad de una orden judicial específica para el retiro del dicho contenido. Durante las discusiones, es posible identificar que, a pesar de que existe una fuerte apelación a la libertad de expresión, hay otros intereses en disputa, principalmente los de los grandes intermediarios de internet, en la actualidad responsables de la gran 
mayoría de las disputas sobre el contenido generado por otros.

La sistemática que proviene de la inimputabilidad de la web como un principio, trata genéricamente a todo tipo de proveedores y no intenta ni agradar, ni desarrollar compañías, ni la posibilidad técnica de control que pueden ejercer por los filtros de los administrados.

Respecto al análisis de la contradicción, entre el discurso de los programadores de software acerca de la necesidad de protección de los derechos individuales y la gran aglutinación de poder que el control de los medios tecnológicos ha proporcionado, Pariser (2012) argumenta: "Si como en la famosa declaración de Lessig (1999), el código es la ley, es importante para nosotros poder entender lo que los nuevos legisladores tienen en mente, y lo que los programadores de Google y Facebook creen» (p.23), En otra parte, él es enfático cuando dice que él cree que son las verdaderas intenciones de los grandes intermediarios cuando profesan una libertad absoluta y sin restricciones como la base de sus conductas en la Web, al respecto sostiene:

Los ejecutivos de Facebook, Google y otras empresas socialmente importantes se engañan a sí mismos: y están involucrados en revoluciones sociales cuando se sabe que es bueno para ellos y los hombres de negocios inmorales o cuando que no lo es. $Y$ ambos comportamientos vienen muy de la mano con lo que se espera (Pariser, 2012:156).

Estas afirmaciones, aunque están basadas en las declaraciones específicas de diversos profesionales del tema, aún carecen de comprobación y, si son ciertas, podrían ser contorneadas cuando se toma a la realidad nacional. Sobre todo si el escrito propuesto en el sustitutivo de la Comisión Especial de la Cámara de Diputados es aprobado.

Después de todo, ambas reservas expresadas en el cuerpo del artículo 15 podrán ser orientadas a las futuras obras hermenéuticas sobre el tema. La primera es sobre la mención que expresa la garantía de la libertad de expresión y la segunda es la frase final que pone de relieve la posibilidad de la coexistencia con otros sistemas de responsabilidad en diferentes leyes. Los valores que sopesan, o que le dan a la libertad una expresión de más peso, en abstracto y sin pérdida a otros valores igualmente pertinentes de la Ley que pueda prevalecer en los casos, son muy importantes para el intérprete. Valores como la tutela de los aspectos de la personalidad, tales como imágenes, atributo, privacidad y otros, no pueden ser simplemente ignorados por la web.

Por lo tanto, la importancia para el Parlamento radica en fijar los intereses en disputa en la elaboración de la redacción de la ley. Como creación de un sistema de responsabilidad que supone que no hay ninguna responsabilidad por cualquier desprecio, haciendo que exista un deber de eliminar lo que dependen de la provisión judicial específica sobre el lugar exacto de la información, desprotegiendo el eslabón más débil de esta cadena: el usuario.

Utilizando el carácter absoluto de la libertad de expresión como un escape para proteger los modelos empresariales irresponsables, parece ser que la subversión está completa con los valores constitucionales, que siempre tiene las situaciones subjetivas existenciales como el corolario del epicentro axiológico de la Ley: la dignidad humana. En otras palabras, utilizando el derecho fundamental a la libertad de expresión, como una base para la «inimputabilidad» de cada intermediario de la web; oculta la tutela de un único derecho fundamental, en detrimento de todos los demás: la libre empresa.

Es por esto, que a pesar de que existe una gran participación popular, en la experiencia del marco brasileño de los derechos civiles para los usuarios de la web todavía no parece haber una personalización de la muerte profesada por el Parlamento, pero sólo la evidencia de una de sus muchas funciones constitucionales, en el presente caso podrá promover la participación popular como un enriquecimiento y fortalecimiento de los elementos de deliberación. 


\section{CONCLUSIONES}

Las TIC han introducido profundas y significativas alteraciones sociales. A medida que están más acostumbrados, parece que cada vez más se están convirtiendo en un camino sin regreso, cuyas consecuencias deben ser medidas.

Este artículo tuvo como objetivo conocer más sobre un aspecto problemático, que es la capacidad del ambiente político creado por el uso masivo de las TIC, especialmente la internet, que se considera un espacio público o una esfera pública en todo el mundo.

En primer lugar, se trató de trabajar algunos aspectos estructurales del concepto de esfera pública, su importancia para la teoría democrática y las críticas a sus supuestos. Luego, se trabajó el problema de la viabilidad de la web al obtener una consideración de herramientas para promover y desarrollar una esfera pública en todo el mundo. Finalmente, se concluyó que hoy en día la web, ha evolucionado a pasos agigantados. Por lo tanto, para que sea una herramienta de promoción eficaz de los supuestos democráticos, y naturalmente de la consecución de una esfera pública en todo el mundo, es necesario dirigir la mirada al escepticismo, pero sin una actitud ciega del optimismo.

Por lo tanto, es necesario hacer un balance de las peculiaridades de la web 2.0, cuya actuación de los filtros de búsqueda, en un entorno de la información «personalizada» de acuerdo a las preferencias del usuario, se desarrolla de acuerdo a los intereses de promoción de productos y servicios, lo cual trae a la democracia aspectos que pueden ser significativamente negativos.

Después de describir este entorno, es posible hacer frente a algunas perspectivas para la representación política, y en concreto a la parte del Legislativo, que se materializó en el Parlamento. Se debe prestar atención al proceso legislativo, pues dice que no es una unanimidad el decir que la tecnología sin duda dará lugar a la consolidación de la democracia directa. Al menos en corto tiempo.

Por tanto, algunos sostienen incluso que la posibilidad de manifestaciones directas de la voluntad política, por parte de la población, no será posible si no necesita la representación, que será capaz de utilizar las tecnologías, pero que no va a desaparecer. $Y$ hay quienes dicen que ya hay un movimiento creciente de la utilización de estas tecnologías por parte del propio legislativo para promover un ambiente que hace que el Parlamento sea un entorno más interactivo y legislativo en un proceso colaborativo.

Por último, es posible identificar a los que dicen que este ambiente dirigirá al poder legislativo a perder su función como protagonista de su proceso, en el ejercicio de otras funciones atípicas, que pueden hacer un fortalecimiento por el uso masivo de las TIC.

No hay una respuesta, pero lo cierto es que en realidad el problema está ahí y la única certeza que existe es que es necesario estudiar a profundidad las potencialidades de la web, así como los retos para su promoción y desarrollo, que pasa por la imposición de límites efectivos a los intereses que pueden matar la esperanza de un futuro más democrático.

\section{REFERENCIAS BIBLIOGRÁFICAS}

- Barros, A. (2008). La importancia del concepto de la esfera pública de Habermas para el análisis de la prensa - un tema de opinión. Universitas 5n. 1/2, p. 23-34, Archit. y Com. Sociales, Brasilia.

- Bauman, Z. (2013). Daños colaterales: las desigualdades sociales en la era global. Trad.: Carlos Alberto Medeiros. Río de Janeiro: Zahar.

- Becak, R; Longhi, J. (2011). Tendencias de la democracia participativa: la influencia de Internet en el perfil de la representación y eventos del presupuesto participativo. En: XX Congreso Nacional CONPEDI. Vitória. La Orden Jurídico Justa: Un Diálogo Americano Europeo.

- Becak, R. (2008). La hipertrofia del Ejecutivo brasileño: el impacto de la constitución de 1988. Campinas: Milenio

- Becak, R. (2011). El Estado de derecho, las formas de Estado y la Constitución. Revista de la Facultad de Derecho de Conselheiro Lafaiete. Nueva fase. v. 3, 2007a. p. 293-295. 
- Becak, R. (2012). Reflexiones sobre la democrática evolucionan hacia su optimización: la realidad de la «democracia deliberativa» y el apoyo teórico. Enfoque histórico-evolutivo. Contribución a la Teoría General del Estado. 178 f. Tesis. Facultad de Derecho de la USP, São Paulo

- Becak, R; (2011). La democracia deliberativa y la ciberdemocracia: riesgos y retos para su implementación. Actas de la «XXIII Congreso Nacional de CONPEDI». Consejo Superior de Investigaciones Científicas. São Paulo.

- $\quad$ Becak, R; Longhi, J. (2012). Tendencias de la democracia participativa: la influencia de internet en el perfil de la representación política y en el Presupuesto Participativo. En: Derecho y Sociedad Encuentro Internacional Sindical, Honolulu. Conversaciones sociojurídicos a través de un mar de los océanos.

- Becak, R, Longhi, J. (2012). Proceso legislativo colaborativo: la participación de la web en la tramitación del proyecto de ley n. 2126/2011 (Marco Civil de Internet). Publicado en los anales de la «XXI Congreso Nacional de CONPEDI», celebrada del 31 oct-3 nov, 2012, en Niterói - RJ.

- Bernardes, M. (2013). La democracia en la Sociedad de la Información: el desarrollo de la democracia digital en los municipios. São Paulo: Saraiva.

- $\quad$ Bobbio, N; Mateucci, N; Pasquino, G. (1998). Diccionario Político. Traducción C. Carmen Varriale otra. Coord. trad. Ferreira, F; Rev. General Ferreira, J; Guerrero, L. 11. ed. Brasilia: Editora Universidad de Brasilia.

- Bonavides, P. (2001). La teoría constitucional de la democracia participativa. São Paulo: Malheiros.

- Caggiano, M; Salem, H. (2011). Notas de la clase dictada en la disciplina «derecho parlamentario», que figuran en el curso de Postgrado Facultad de Derecho de la Universidad de São Paulo, junto con el Prof. Dr. Rubens Beçak en el segundo semestre de 2011.

- Carvalho, K. (2009). Derecho Constitucional: Teoría del Estado y de la Constitución; derecho constitucional positivo. Ed 15a. Rev., actualizada y ampl. Belo Horizonte: Del Rey.
- Hawk, J. (2006). La democracia, la ley y el tercer sector. 2. ed. Río de Janeiro: FGV.

- Castells, M. (2013). Redes de indignación y esperanza. Río de Janeiro: Zahar.

- Garza, J. (1997). Derecho Parlamentario. México: UNAM.

- Gomes, W, Rosely, M. (2008). Comunicación y democracia. problemas y perspectivas. São Paulo: Paulus.

- Habermas, J. (1997). Derecho y democracia: entre facticidad y validez. Vol. II. Trad. Fabio Beno Siebneichler. Río de Janeiro: Tiempo de Brasil.

- Hindman, M. (2009). El mito de la democracia digital. Princeton: Princeton University Press.

- Lemos, A; Levy, P. (2010). El futuro del internet: hacia una ciberdemocracia planetaria. São Paulo: Paulus.

- Lessig, L. (1999). Código y otras leyes del ciberespacio. Nueva York: Basic Books.

- Longhi, J; Rozatti, V. (2009). Sistemas y teoría de Niklas Luhmann y el derecho a la información en la legislación brasileña. El «robo» de la domesticación jurídico real de camellos en la ley de propiedad intelectual en Internet. Artículo publicado en el XVIII Congreso Nacional CONPEDI. São Paulo, 2009.

- Malberg, R. (2001).Teoría general del Estado. Trad. José Lión Depetre. México: Fondo de Cultura Económica.

- $\quad$ Mendes, G; Conejo, M; Blanco, P. (2009). Curso de derecho constitucional. São Paulo: Saraiva.

- Pariser, E. (2012). El filtro invisible. Lo que la Internet está escondiendo de ti. Trad. Diego Alfaro. Río de Janeiro: Zahar.

- Perez, A. (2002). Internet y Derechos Humanos. Derecho y Conocimiento: Anuario Jurídico Sobre la Sociedad de la Información y del Conocimiento de la Universidad de Huelva (Facultad de Derecho), Huelva, No. 2, pp. 101-121.

- Rodotà, S. (2008). La vida en la sociedad de la vigilancia: la privacidad en la actualidad. Organización, selección y presentación de María Celina Bodin de Moraes. Traducción Danilo Doneda y Luciana Cabral Doneda. Río de Janeiro: Actualizar.

- Zagrebelsky, G. (2011). La crucifixión y la democracia. São Paulo: Saraiva. 


\section{PÁGINAS WEB}

- O'Reilly, T. (2005). ¿Qué es la Web 2.0. Patrones del diseño y modelos del negocio para la siguiente generación de software. Disponible en: http://oreilly.com/web2/archive/what-is-web20.html. Consultado el: 08 de febrero 2014.

- Rover. J; Mezzaroba, O. (2011). Nuevas tecnologías: el gobierno electrónico desde la perspectiva de la gobernanza. En: (Org) Vladimir Oliveira da Silveira y Orides Mezzaroba. Compañia, la sostenibilidad y la funcionalización del derecho. - Sao Paulo: Editora Revista dos Tribunales, (Justicia colección, Empresa y Sostenibilidad; v.2). Disponible en: http:// www.egov.ufsc.br/portal/sites/default/files/ novas_tecnologias_-_uninove.pdf. Acceso en: 23 de agosto 2012.

- Silva, J. (2002). El sistema representativo y la democracia semi-directa: la democracia participativa. En: sistema de la democracia representativa Cantú, Hugo A. y semidirecta. VII Memorial del Congreso de Derecho Constitucional. México: Instituto de Investigaciones Jurídica de la Universidad Autónoma de México.

- Trein, A. (2013). Ciberdadania y República.com @: reflexiones desde Cass Sunstein y Pérez Luno. Revista Digital y Electrónica Democracia Gobierno, No. 9, p. 1-23. Disponible: http:// buscalegis.ufsc.br/revistas/index.php/ observatoriodoegov/article/view/34274. Acceso: 31 de enero 2014.

- Vaidhyanathan, S. (2012). Globalización de todo (y por qué no nos debería preocupar). Berkeley / Los Angeles: University of California Press.

- Castardo, H. (2007). Naturaleza jurídica del Tribunal de Cuentas en el Sistema Legal de Brasil. 2007. 190 f. Tesis (Maestría en Derecho) - Facultad de Derecho de la Universidad Metodista de Piracicaba, Piracicaba. Director: Sergio Resende de Barros. Disponible en: https://www.unimep.br/ phpg/bibdig/pdfs/2006/YTAVWAIQBHIT.pdf. Acceso el 1 de febrero 2014.

- Castells, M. (2008). La Nueva Esfera Pública: la Sociedad Civil Global, redes de comunicación, y la gobernanza global. En: Anales de la Academia Americana. Disponible en:
- http://annenberg.usc.edu/Faculty/ Communication\%20and\%20Journalism/ /media/ 78.ashx. Acceso el 27 de enero 2014.

- Clarke, R; Morell, M; Piedra, G; Sustein, C; Swire, P. (2013). Libertad y seguridad en un mundo cambiante: Informe y Recomendaciones del Informe y Recomendaciones del Grupo de Revisión del Presidente sobre Inteligencia y tecnologías de comunicaciones. -Disponible en: http://s3.documentcloud.org/documents/929267/ review- grupo exec-summary-y-recs.pdf. Acceso el 26 de enero 2014.

- Dahl, R. (2012). La democracia y sus críticos. Trad. Patricia de Freitas Ribeiro; Rev. de trans. Aníbal Mari. São Paulo: Martins Fontes WMF.

- Faria, F. (2012). El parlamento abierto en la era de Internet: las personas pueden trabajar con la Legislatura en la elaboración de las leyes? - Brasilia: Cámara de Diputados, ediciones Cámara. Disponible en: http://bd.camara.gov.br/bd/ bitstream/handle/bdcamara 7867 / parlamento_aberto_faria.pdf? secuencia $=2$. Acceso: 01 de febrero 2014.

- Longhi, J. Rozatti, V. (2013). Privacidad, democracia y redes sociales en Brasil: ¿de invierno primavera? - Medialaws. Disponible en: http:// www.medialaws.eu/privacidad-democracia-y-redes-sociales-en-brasil-primavera-o-inverno/. Acceso: 27 Enero 2014

- Congreso Nacional - Cámara de Representantes. El Informe de la Comisión Especial diseñado para emitir un dictamen sobre el Proyecto de Ley № 2126 de 2011, enviado al Congreso por el Poder Ejecutivo por Mensaje № 326 de 2011. Disponible en: http://edemocracia.camara.gov. br / documentos / 679637 / 277cc749-e543-4636$9 \mathrm{ddb}-736144 \mathrm{a} 9 \mathrm{~b} 654$. Acceso en: 30 de agosto 2012.

- Amaral, R. (2001). Notas para la reforma política: la democracia representativa ha muerto; viva la democracia participativa. Revista de Información Legislativa. n. 151. Brasilia. Disponible en: http://bd.camara.gov.br/bd/bitstream/ secuencia de mango/bdcamara /461/ analise_partidario_ lima.pdf = 8 Consultado Febrero 2014. 